\title{
Los recursos naturales de la finca El Morro, San Miguelito, Río San Juan, Nicaragua, 2013
}

\author{
El Morro Farm 's natural resources, San Miguelito, Río San Juan, Nicaragua, 2013
}

\author{
Fredy Leonel Valiente Torres ${ }^{1}$ \\ Claudio Calero ${ }^{2}$
}

\begin{abstract}
Resumen
Este estudio realizado en la finca El Morro, municipio de San Miguelito, departamento de Río San Juan, Nicaragua. Fueron evaluadas las características biofísicas de los recursos bosque, fauna silvestre, agua y suelo. Fue inventariado con diseño de muestreo sistemático de acuerdo a los diferentes tipos de cobertura vegetal, tales como: bosque abierto, tacotal y árboles dispersos en potrero. Se ubicaron dos parcelas circulares, con un radio de 15 metros, separadas a una distancia de tres $\mathrm{km}$ entre ellas, la fauna silvestre se hizo simultánea al levantamiento de la información florística. En este estudio se trata de conocer la riqueza florística y el estado silvicultural de la vegetación arbórea, la fauna existente, la calidad del agua y caracterización del suelo, con la finalidad de contribuir a la toma de decisiones en la implementación de buenas prácticas para el manejo de todos los recursos existentes en la finca.
\end{abstract}

Palabras clave: Diagnóstico; conservación; bosque abierto; tacotal; árboles en potreros; suelo; calidad de agua; fauna silvestre.

\begin{abstract}
This study was carried out at El Morro farm, San Miguelito municipality, Río San Juan department, Nicaragua. The biophysical characteristics of the forest, wildlife, water and soil resources were evaluated. It was inventoried with a systematic sampling design according to the different types of vegetation cover, such as: open forest, tacotal (which is defined as uncultivated land covered with thick undergrowth) and scattered trees in pasture. Two circular plots were located, with a radius of 15 meters, separated at a distance of three $\mathrm{km}$ between them, the wild fauna was done simultaneously with the collection of floristic information. This study tries to know the floristic richness and the silvicultural state of the arboreal vegetation, the existing fauna, the quality of the water and the characterization of the soil, in order to contribute to decision-making in the implementation of good practices for the management of all existing resources on the farm.
\end{abstract}

Keywords: Diagnosis; conservation; open forest; tacotal; trees in paddocks; ground; water quality; wildlife

\section{Introducción}

Nicaragua es un país privilegiado por sus enormes riquezas naturales, es por eso que la protección y conservación de sus recursos naturales requiere de acciones concretas y de un trabajo conjunto de técnicos y productores. En ese sentido, el manejo de los bosques naturales es importante en la protección y conservación de los ecosistemas naturales. 
En la finca El Morro, ubicada en el municipio de San Miguelito, departamento de Río San Juan existe vegetación forestal y ambiente lacustre, con la presencia de dos clases de aves, las presentes dentro del área de bosque y a lo largo de la costa del lago Cocibolca, lo que indica que los ecosistemas existentes en la finca influyen en el tipo de fauna silvestre, sobre todo de aves en la zona (Calero, 2016).

Se tomaron 20 muestras de suelo en cada tipo de cobertura vegetal para determinar el estado físico y químico. Se evaluó la calidad del agua para consumo humano en las dos fuentes hídricas existentes. En bosque abierto se encontraron29 especies y 18 familias. Las familias con más individuos fueron: Bignoniaceae, Caesalpinaceae, Fabaceae y Mimosaceae; en el tacotal se encontraron 26 especies y 19 familias, sobresaliendo en número de individuos, Tiliaceae, Bixaceae, Sterculiaceae y Mimosaceae; en el área de árboles dispersos potreros se registraron 34 especies y 21 familias, siendo Anarcadiaceae, Fabaceae, Mimosaceae y Sterculiaceae las de mayor abundancia de individuos. El índice de diversidad de Shannon-Wiener indica que todos los tipos de vegetación tienden a la heterogeneidad o equitatividad en especies e individuos. En los tres estratos se encontraron 26 especies de aves, 7 de mamíferos y 8 de reptiles. Se evaluó el pH, nitrito, nitrato, amonio, fosfato, dureza de carbono, dureza total y oxígeno del agua, encontrando que es apta para consumo humano; sin embargo, no se hizo análisis biológico, por tanto, se desconoce la concentración de coliformes fecales de las dos fuentes. La textura de los suelos en los tres tipos de vegetación es arcillosa.

En general el bosque de la finca se ha deteriorado por diferentes actividades agropecuarias, lo que ha ocasionado que la fauna emigre por la pérdida de su hábitat natural, deterioro de las fuentes hídricas, erosión y pérdida de suelo (Calero, 2016).

Las buenas prácticas que se puedan aplicar en la finca contribuirían a la conservación del sistema de humedales de San Miguelito, catalogados sitio RAMSAR 1140, importante ecosistema que alberga diversidad de especies de fauna lacustre (Ruiz, 2003).

\section{Materiales y métodos}

\section{Descripción del sitio de estudio}

El estudio se desarrolló en la finca El Morro, municipio de San Miguelito, departamento de Río San Juan, ubicado a $254 \mathrm{~km}$ de Managua en el 2013. La principal actividad fue levantamiento de información sobre los factores edáficos, afluentes de agua, riqueza de fauna y diversidad florística de la finca, lo que se convirtió en la etapa de campo para realizar un diagnóstico.

\subsubsection{Localización geográfica}

La finca está ubicada a $4 \mathrm{~km}$ de la cabecera municipal de San Miguelito (figura 1), con un área total de 337.25 hectáreas, en las siguientes coordenadas N. 729689 W. 1260015, el área de bosque es de 60.76 ha, con suelos franco arcillosos, la precipitación pluvial varía entre los 2000 y $2400 \mathrm{~mm}$ y la temperatura media oscila entre los 25 y $26^{\circ} \mathrm{C}$ (INIFOM, 2010). La vía de acceso a la finca es un camino de tierra en mal estado, no hay acceso en vehículo terrestre, solo a pie o por vía acuática.

\subsubsection{Geomorfología}

El municipio se caracteriza por ser montañoso, con excepción de la franja costera en el margen oriental del gran lago de Nicaragua. En materia hidrográfica el municipio cuenta con los ríos Tepenaguazapa, Tule, Coco, Fajardito, Sapote, Jícaro, Guayabal, Camastro, Tulito y alrededor de 20 quebradas grandes (INIFOM, 2010). Según el mapa de órdenes de suelos de Nicaragua, los suelos de la finca están dentro de la clasificación de vertisoles (INETER, 2002). 


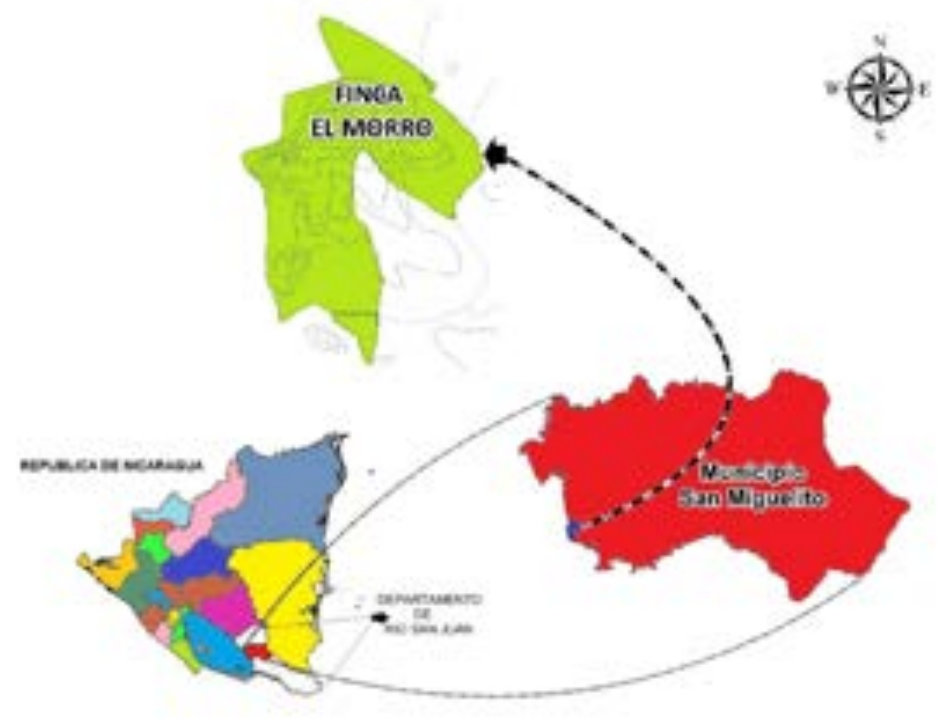

Figura 1. Ubicación de la finca El Morro, San Miguelito, departamento de Río San Juan.

\subsection{Proceso metodológico}

\subsubsection{Levantamiento de la poligonal de la finca y elaboración de mapa base}

Se hizo un recorrido preliminar siguiendo 16 linderos de la finca para reconocer su forma y ubicación de áreas de vegetación existentes, identificando tres estratos: bosque abierto, tacotal y árboles en potreros, posteriormente se elaboró un mapa base con los datos de coordenadas levantados con GPS y usando el programa ArcGIS 10.2.2 (figura 2).

\subsubsection{Inventario florístico}

\section{a) Diseño del inventario}

Se realizó un inventario forestal en cada estrato, mediante un muestreo sistemático (figura 2).

En el bosque abierto, se establecieron tres líneas de inventario, tomando como punto de referencia el inicio del bosque; dos líneas se ubicaron paralelas, distanciadas 100 metros entre ellas, un azimut de 75 grados y una longitud de 700 y 800 metros respectivamente, se establecieron 7 parcelas de 10 x 60 metros en cada una; la otra línea se ubicó con azimut 180 grados y longitud de 900 metros, estableciendo 8 parcelas de las mismas dimensiones, para un total de 22 parcelas, un área de muestreo de 1.32 ha y una intensidad de muestreo de $2.17 \%$.

En el tacotal se establecieron tres líneas de inventario paralelas, separadas 100 metros entre ellas, con una longitud de 300 metros, a partir de un punto de referencia dado por la accesibilidad de las pendientes prevalecientes en el sitio. Sobre cada línea se establecieron tres parcelas de $10 \mathrm{x} 60$ metros, distanciadas 100 metros entre ellas, para un total de nueve parcelas, un área de muestreo de 0.54 ha y una intensidad de muestreo de $6.41 \%$. 
En ambos tipos de estratos, dentro de cada parcela se midieron todos los árboles a partir de 10 centímetros de diámetro normal (DN). En el estrato árboles en potrero se estableció un área cuya línea base fue el cerco que limita con la finca vecina, donde se establecieron 9 líneas de inventario, con longitud de 700 $\mathrm{m}$ cada una, separadas $100 \mathrm{~m}$ entre ellas; en cada línea se establecieron 7 parcelas de $10 x 10 \mathrm{~m}$, separadas $100 \mathrm{~m}$. En una décima línea, por la naturaleza del terreno, solo se establecieron 4 parcelas. En una segunda área se establecieron tres líneas de inventario de $300 \mathrm{~m}$, separadas $100 \mathrm{~m}$, ubicando tres parcelas de $10 \times 10 \mathrm{~m}$, separadas por $100 \mathrm{~m}$. En total, se establecieron 76 parcelas, un área de muestreo de 0.76 ha y una intensidad de muestreo de $0.81 \%$. Las parcelas fueron pequeñas porque sólo se contabilizaron individuos de la regeneración natural.

\section{b) Medición de variables}

En el bosque abierto y en el tacotal se evaluaron los árboles con diámetro mayor a $10 \mathrm{~cm}$, midiendo en cada árbol su diámetro normal a una altura de $1.30 \mathrm{~m}$ del suelo, utilizando cinta diamétrica; además, se estimó la altura total del árbol en pie, utilizando un clinómetro Suunto (Anexo 1). Los nombres comunes de los árboles se obtuvieron con los baquianos del sitio y para obtener sus nombres científicos se consultó el libro Árboles de Nicaragua (Salas, 1993).

\section{c) Índice de Shannon-Wiener}

El índice de Shannon-Wiener mide el grado promedio de incertidumbre y predice a qué especie puede pertenecer un individuo escogido al azar, asumiendo que los individuos son seleccionados al azar y que todas las especies están representadas en una muestra (Narváez, 2012), analizando la riqueza y diversidad florística de la vegetación, (Orozco, 1991).

Su fórmula es: $H^{\prime}=-\Sigma^{s}\left[(n i / n)^{*} \ln (n i / n)\right]$

$$
i=1 \text {, donde: }
$$

$H^{\prime}=$ Índice de Shannon - Wiener

$n i=$ Número de individuos que pertenece a la $\mathrm{i}$ - esima especies en la muestra $\mathrm{n}=$ Número total de individuos en la muestra

El índice se utilizó en este estudio para determinar la diversidad de especies en los tres estratos, siendo válido sólo dentro de cada uno y no comparable entre ellos.

Figura 2. Diseño del inventario por muestreo sistemático, en la finca El Morro, 2013.

\subsubsection{Reconocimiento de fauna silvestre}

En el bosque abierto se ubicaron dos parcelas circulares de 15 metros de radio, separadas $3 \mathrm{~km}$ entre ellas, tomando como criterio la mayor cantidad de vegetación arbórea. En cada parcela, en dos semanas consecutivas, durante 15 minutos, se hizo reconocimiento de aves por sus cantos, sonidos y observación directa. También se observaron mamíferos y reptiles, además de la identificación de huellas, excrementos, madrigueras, nidos y restos orgánicos, auxiliándose de guías ilustradas (Dean, 2011) y con la experiencia y ayuda de los baquianos.

En las áreas de tacotal y árboles dispersos en potrero el reconocimiento de la fauna se hizo en las líneas y parcelas del inventario, para mayor facilidad, simultáneo al levantamiento de la información florística. Las líneas de inventario se tomaron como transeptos (figura 3). Además de lo observado en el campo, se tomó en cuenta la información proporcionada por los baquianos acerca de cuáles especies de fauna han 
observado en el lugar. También se obtuvo información adicional por medio de entrevistas informales a dos pobladores locales.

Con la información recolectada se elaboró un listado de las especies observadas y referidas y se utilizó el instrumento de la clasificación de fauna, como criterio de su estado de conservación, según el convenio Convención sobre el Comercio Internacional de Especies Amenazadas de Fauna y Flora Silvestre (CITES, 2010).

\section{a) Clasificación de fauna según apéndice de CITES}

La CITES es un acuerdo internacional cuya finalidad es velar que el comercio internacional de especies de animales y plantas silvestres no constituya una amenaza para su sobrevivencia, sometiendo al comercio internacional de especies a un determinado control. Toda importación, exportación, reexportación o introducción de especies amparadas por la CITES debe autorizarse mediante un sistema de concesión de permiso o certificado (CITES, 2010). Las especies amparadas por CITES están incluidas en tres apéndices según el grado de protección que necesitan:

Apéndice I: Especies en peligro de extinción. El comercio de especies de este grado está prohibido y se autoriza solamente bajo circunstancias excepcionales.

Apéndice II: Especies no necesariamente en peligro de extinción, pero su comercio debe controlarse para evitar una utilización incompatible con su supervivencia.

Apéndice III: Especies que están protegidas al menos en un país, que ha solicitado la asistencia de otras partes de CITES para control de su comercio (CITES, 2010).

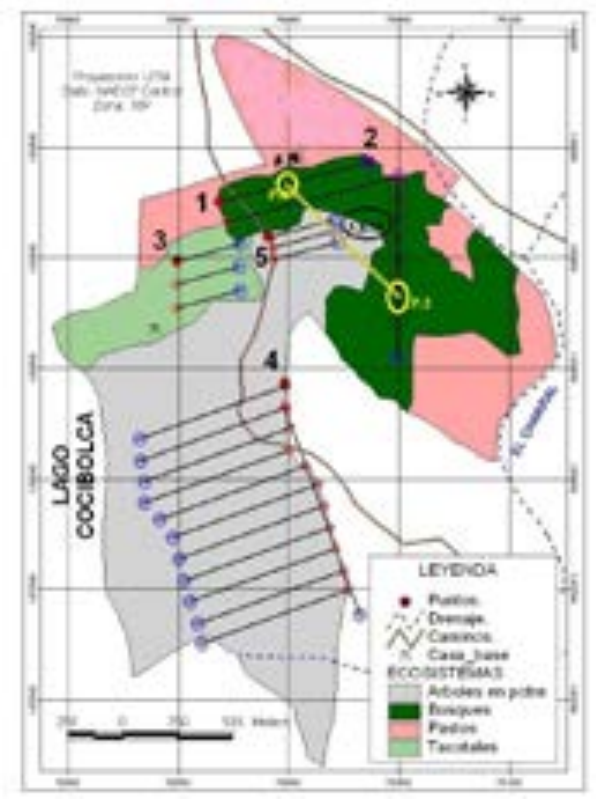

Figura 3. Diseño del inventario faunístico en parcelas circulares, en la finca El Morro, 2013.

\subsubsection{Caracterización de suelos}

Se perforaron tres semi-calicatas en los tres estratos para observar la profundidad y número de horizontes del suelo, en cada uno de los tipos. Para la caracterización se tomaron 20 sub-muestras de suelo en cada estrato, para un total de 6o sub-muestras que se llevaron al Laboratorio de Suelos de la Universidad 
Nacional Agraria, con el fin de determinar su estructura física (partículas de arcilla, limo, arena, para la textura) y composición química (pH, Materia orgánica, N, P-disp., CE, K-disp. Al) (USDA, 1999).

\subsubsection{Análisis de calidad de agua}

Se realizó una evaluación de la calidad del agua para consumo humano en las dos fuentes hídricas existentes en la finca, que son nacimientos de agua natural. Cabe aclarar que estas fuentes de agua están desprovistas de vegetación y que en la ribera de las fuentes se encontraban helechos en un $40 \%$.

Para esta evaluación se utilizó un kit de campo de análisis de agua (Agua-Merck, S/f), siguiendo las instrucciones de la guía respectiva, donde se determinó concentración de: amonio, nitratos, nitritos, dureza de carbono, dureza total, $\mathrm{PH}$, fosfato y oxígeno (Anexo 3).

\section{Resultados y discusión}

\subsection{Composición florística y diversidad de especies arbóreas y arbustivas}

\subsubsection{Bosque abierto}

En este estrato se encontraron 156 árboles, en un área de muestreo de 1.32 ha, para una densidad de 120 árboles/ha. Se identificaron 29 especies agrupadas en 18 familias. Las familias más representativas fueron Bignoniaceae, Caesalpiniaceae, Fabaceae y Mimosaceae, en vista de que fueron las que reportaron mayor número de individuos durante el inventario (cuadro 1). La vegetación en este estrato consiste en una sucesión secundaria joven, evidenciada por las distribuciones diamétrica encontradas, ya que la mayoría de los individuos no sobrepasan los 30 centímetros de diámetro, el cual se está regenerando con árboles de bajo valor comercial como Cecropia peltata, Apeiba membranacea y Guazuma ulmifolia. Durante el muestreo en este estrato se encontraron cuatro claros, lo que muestra que a pesar de que es un bosque joven este se sigue interviniendo, con actividades como extracción de leña y madera para construcción de botes, así como el cambio de uso de la tierra para ganadería.

\subsubsection{Tacotal}

En el tacotal se registraron 73 individuos, agrupados en 26 especies y 19 familias. Las familias más comunes fueron Tiliaceae, Bixaceae, Sterculiaceae y Mimosaceae (cuadro 2). De las 26 especies, el 44\% (12 especies) son maderables, lo que indica que el uso maderable tiene preferencia por la población sobre todo como madera de construcciones rurales, a pesar de las intervenciones en el cambio de uso del suelo para la actividad ganadera que se le dio a la finca.

Cuadro 1. Especies en el estrato bosque abierto de la finca El Morro, 2013.

\begin{tabular}{|l|l|l|l|l|}
\hline $\mathbf{N}^{\circ}$ & \multicolumn{1}{|c|}{ Nombre común } & \multicolumn{1}{|c|}{ Nombre científico } & \multicolumn{1}{c|}{ Familia } & \multicolumn{1}{c|}{ Ind./especie } \\
\hline 1 & Jocote jobo & Spondias mombin L. & Anarcadiaceae & 18 \\
\hline 2 & Falso roble & Tabebuia rosea Bertol. & Bignoniaceae & 2 \\
\hline 3 & Cortez & Tabebuia crysantha Jacq & Bignoniaceae & 2 \\
\hline 4 & Poro poro & Cochlospermum vitifolium Willd. & Bixaceae & 6 \\
\hline 5 & Ceiba & Ceiba aesculifolia Kunth. & Bombacaceae & 3 \\
\hline 6 & Muñeco & Cordia collococca L. & Boraginaceae & 2 \\
\hline 7 & Jiñocuao & Bursera simarouba L. & Burseraceae & 7 \\
\hline 8 & Malinche & Delonix regia Bojer ex Hoot. & Caesalpiniaceae & 1 \\
\hline 9 & Comenegro & Dialum guianensis L. & Caesalpiniaceae & 1 \\
\hline 10 & Guarumo & Cecropia peltata L. & Cecropiaceae & 32 \\
\hline
\end{tabular}




\begin{tabular}{|c|c|c|c|c|}
\hline $\mathrm{N}^{\circ}$ & Nombre común & Nombre científico & Familia & Ind./especie \\
\hline 11 & Hoja chigua & Curatella americana L. & Dilleniaceae & 3 \\
\hline 12 & Camíbar & Copaifera aromatica Jacq. & Fabaceae & 5 \\
\hline 13 & Guachipilín & Diphysa robinioides Mill. & Fabaceae & 1 \\
\hline 14 & Elequeme & Erythrina fusca Lour. & Fabaceae & 1 \\
\hline 15 & Madero negro & Gliricidia sepium Jacq. & Fabaceae & 6 \\
\hline 16 & Aguacate de monte & Ocotea coeruleum Mez et Donn. & Lauraceae & 2 \\
\hline 17 & Capirote & Miconia argentea Cogn. & Malastomaceae & 1 \\
\hline 18 & Nancite & Byrsonima crassifolia L. & Malpighiaceae & 5 \\
\hline 19 & Guanacaste blanco & Albizzia caribaea Urb. & Mimosaceae & 1 \\
\hline 20 & Gavilán & Albizzia guachapele Dugan. & Mimosaceae & 2 \\
\hline 21 & Guanacaste negro & Enterolobium cyclocarpum Jacq. & Mimosaceae & 1 \\
\hline 22 & Guaba & Inga densifolia L. & Mimosaceae & 1 \\
\hline 23 & Gavillo & Inga vera Mart. & Mimosaceae & 2 \\
\hline 24 & Genízaro & Pithecelobium saman Jacq. & Mimosaceae & 8 \\
\hline 25 & Guácimo ternero & Guazuma ulmifolia Lam. & Sterculiaceae & 16 \\
\hline 26 & Panamá & Sterculia apetala Jacq. & Sterculiaceae & 7 \\
\hline 27 & Peine mico & Apeiba membranacea Spruce ex benth & Tiliaceae & 16 \\
\hline 28 & Capulín negro & Trema micrantha L. Blume & Ulmaceae & 2 \\
\hline \multirow[t]{2}{*}{29} & Melina & Gmelina arbórea Roxb. & Verbenaceae & 2 \\
\hline & & 29 especies & 18 Familias & 156 \\
\hline
\end{tabular}

Cuadro 2. Especies encontradas en el estrato tacotal en la finca El Morro

\begin{tabular}{|l|l|l|l|}
\hline \multicolumn{1}{|c|}{ Nombre común } & \multicolumn{1}{|c|}{ Nombre científico } & \multicolumn{1}{c|}{ Ind/especie } \\
\hline Jocote jobo & Spondias mombin L. & Anarcadiaceae & 1 \\
\hline Falso roble & Tabebuia rosea Bertol. & Bignoniaceae & 2 \\
\hline Achiote silvestre & Bixa orellana L. & Bixaceae & 1 \\
\hline Poro poro & Cochlospermum vitifolium Willd. & Bixaceae & 2 \\
\hline Ceiba & Ceiba aesculifolia Kunth & Bombacacea & 1 \\
\hline Laurel hembra & Cordia alliodora Ruiz \& Pavón & Boraginaceae & 3 \\
\hline Guarumo & Cecropia peltata & Cecropiaceae & 1 \\
\hline Hoja chigua & Curatela americana & Dilleniaceae & 11 \\
\hline Granadillo & Dalbergia tucurensis Donn. Sm. & Fabaceae & 1 \\
\hline Guachipilín & Diphysa robinioides Mill. & Fabaceae & 2 \\
\hline Aguacate montero & Ocotea coeruleum Mill. & Lauraceae & 6 \\
\hline Capirote & Miconia argentea Cogn. & Malastomaceae & 7 \\
\hline Nancite & Byrsonima crassifolia & Malpighiaceae & 7 \\
\hline Piojo & Trichilia americana Seseé \& Moc & Meliaceae & 1 \\
\hline Guanacaste negro & Enterolobium cyclocarpum Jacq. & Mimosaceae & 3 \\
\hline Gavillo & Inga vera Mart. & Mimosaceae & 2 \\
\hline Genízaro & Pithecelobium saman Jacq & Mimosaceae & 2 \\
\hline
\end{tabular}




\begin{tabular}{|l|l|l|l|}
\hline \multicolumn{1}{|c|}{ Nombre común } & \multicolumn{1}{|c|}{ Nombre científico } & \multicolumn{1}{c|}{ Familia } & \multicolumn{1}{c|}{ Ind/especie } \\
\hline Guayaba & Psidium guajava & Myrtaceae & 4 \\
\hline Espino negro & Pisonia aculeata L. & Nyctaginaceae & 1 \\
\hline Guácimo ternero & Guazuma ulmifolia Lam. & Sterculiaceae & 2 \\
\hline Panamá & Sterculia apetala Jacq. & Sterculiaceae & 1 \\
\hline Burillo & Apeiba tibourbou Aubl. & Tiliaceae & 3 \\
\hline Guácimo de molenillo & Luehea candida Lam. & Tiliaceae & 2 \\
\hline Guácimo colorado & Luehea seemannii Lam. & Tiliaceae & 1 \\
\hline Capulín negro & Trema micrantha Blume & Ulmaceae & 2 \\
\hline Melina & Gmelina arborea Roxb & Verbenaceae & 4 \\
\hline & 26 especies & 19 familias & 73 \\
\hline
\end{tabular}

\subsection{3. Árboles dispersos en potreros}

Se registraron 188 individuos menores de $10 \mathrm{~cm}$ de diámetro, agrupados en 34 especies y 21 familias. Las familias más representativas fueron Anarcadiaceae, Fabaceae, Mimosaceae y Sterculiaceae. En la línea 11 todas las parcelas se establecieron en suelo saturado de agua, no encontrándose ninguna especie forestal (Cuadro 3).

Cuadro 3. Lista de especies en el estrato árboles dispersos en potreros, finca El Morro, San Miguelito, Río San Juan, 2013.

\begin{tabular}{|c|c|c|c|c|}
\hline Nombre común & \multicolumn{2}{|c|}{ Nombre científico } & Familia & Ind./especie \\
\hline Jocote jobo & \multicolumn{2}{|l|}{ Spondias mombin L, } & Anarcadiaceae & 1 \\
\hline Ronrón & \multicolumn{2}{|c|}{ Astronium graveolens Engl. } & Anarcadiaceae & 1 \\
\hline Soncoya & Annona purpurea & MOC. & Annonaceae & 1 \\
\hline Cortez & \multicolumn{2}{|c|}{ Tabebuia crysantha Jacq. } & Bignoniaceae & 2 \\
\hline Poro poro & \multicolumn{2}{|c|}{ Cochlospermum vitifolium Willd. } & Bixaceae & 1 \\
\hline Laurel & \multicolumn{2}{|c|}{ Cordia alliodora Ruiz \& Pavón } & Boraginaceae & 2 \\
\hline Muñeco & \multicolumn{2}{|l|}{ Cordia collococa L. } & Boraginaceae & 6 \\
\hline Guarumo & \multicolumn{2}{|l|}{ Cecropia peltata $\mathrm{L}}$, & Cecropiaceae & 3 \\
\hline Hojachigua & \multicolumn{2}{|l|}{ Curatela Americana } & Dilleniaceae & 35 \\
\hline Cero contil & Senna alata L. & & Fabaceae & 11 \\
\hline Cornizuelo & \multicolumn{2}{|l|}{ Acacia collinsii Saff. } & Fabaceae & 2 \\
\hline Helequeme & \multicolumn{2}{|l|}{ Eritryna fusca Urb. } & Fabaceae & 1 \\
\hline Gasparillo & Erythrina coralloides & D.C. & Fabaceae & 6 \\
\hline Granadillo & \multicolumn{2}{|c|}{ Dalbergia tucurensis Donn. } & Fabaceae & 1 \\
\hline Guachipilín & Diphysa robinioides & MILL. & Fabaceae & 4 \\
\hline Aguacate de monte & \multicolumn{2}{|c|}{ Ocotea coeruleum Mill. } & Lauraceae & 2 \\
\hline Capirote & \multicolumn{2}{|c|}{ Miconia argentea Cogn. } & Malastomaceae & 10 \\
\hline Nancite & \multicolumn{2}{|c|}{ Byrsonima crassifolia L. Kunth } & Malpigiaceae & 4 \\
\hline Cedro real & \multicolumn{2}{|l|}{ Cedrela odorata L. } & Meliaceae & 1 \\
\hline Genízaro & \multicolumn{2}{|c|}{ Pithecelobium saman Jacq. } & Mimosaceae & 6 \\
\hline
\end{tabular}




\begin{tabular}{|c|c|c|c|c|c|c|}
\hline Nombre común & \multicolumn{4}{|c|}{ Nombre científico } & Familia & Ind./especie \\
\hline Guanacaste negro & \multicolumn{4}{|c|}{ Enterolobium cyclocarpum Jacq. } & Mimosaceae & 14 \\
\hline Guavillo & Inga vera & Mart & & & Mimosaceae & 1 \\
\hline Roble & \multicolumn{4}{|c|}{ Tabebuia rosea Bertol } & Mimosaceae & 5 \\
\hline Guayaba & Psidium gu & ajava & $\mathrm{L}$. & & Myrtaceae & 30 \\
\hline Espino blanco & \multicolumn{4}{|c|}{ Crataegus laevigata } & Rosaceae & 2 \\
\hline Flor roja & \multicolumn{4}{|c|}{ Ixora coccíneara Raf. } & Rubiaceae & 1 \\
\hline Majagua & \multicolumn{4}{|c|}{ Genipa americana L, } & Rubiaceae & 1 \\
\hline Juarana & \multicolumn{4}{|c|}{ Paullinia cupana Kunth } & Sapindaceae & 3 \\
\hline Guácimo de ternero & \multicolumn{4}{|c|}{ Guazuma ulmifolia L, } & Sterculiaceae & 6 \\
\hline Panamá & \multicolumn{4}{|c|}{ Sterculia apetala Jacq. } & Sterculiaceae & 2 \\
\hline $\begin{array}{l}\text { Guácimo de mole- } \\
\text { nillo }\end{array}$ & \multicolumn{4}{|c|}{ Luehea candida Mart. } & Tiliaceae & 2 \\
\hline Melina & \multicolumn{4}{|c|}{ Gmelina arbórea Roxb } & Verbenaceae & 17 \\
\hline Teca & \multicolumn{4}{|c|}{\begin{tabular}{l|l} 
Tectonis grandis & $\mathrm{L}$. \\
\end{tabular}} & Verbenaceae & 1 \\
\hline Pimienta silvestre & \multicolumn{4}{|c|}{ Vitex agnus-castus L. } & Lamiaceae & 3 \\
\hline & \multicolumn{4}{|c|}{34 especies } & 21 familias & 188 \\
\hline
\end{tabular}

La diversidad florística es mayor en el potrero, donde se encontró una mayor cantidad de especies, esto se debe a que el área está en un proceso de sucesión y regeneración natural, con poca perturbación ya que en esta área no hay pastoreo por la pendiente bastante pronuncia y porque hay un cerco que limita la entrada del ganado (Figura 4).

Cabe mencionar que en los tres estratos lo que predominan son especies sin ningún valor comercial maderable, como hoja chigüe (Curatela americana), capirote (Miconia argentea) y guayaba (Psidium guajava) lo que supone que hubo una fuerte presión sobre las especies maderables existentes en el pasado, pero si no continúa la presión sobre el recurso puede que en un futuro se puedan establecer bajo un proceso de regeneración natural.

\subsection{4. Índice de ShannonWiener}

Los rangos del índice de Shannon-Wiener van de o. A 3.5, esto se interpreta que de o a 2 se trata de una vegetación más homogénea en número de individuos y especies, mientras que si el valor es mayor de 2 corresponde a una vegetación heterogénea indicando que existe más equidad en la distribución de individuos y especies, es decir, no hay predominancia y por lo tanto hay más diversidad (Pla, 2006). En el cuadro 4 se muestran los índices obtenidos por cada estrato, los que aun cuando no se están comparando refleja claramente la mayor diversidad en el estrato árboles en potreros y la condición heterogénea en todos ellos.

Cuadro 4. Índice de Shannon por cada estrato

\begin{tabular}{|l|l|l|l|}
\hline \multicolumn{1}{|c|}{ Estrato } & \multicolumn{1}{c|}{ Riqueza } & \multicolumn{1}{c|}{ Densidad (arb/ha) } & \multicolumn{1}{c|}{ Indice Shannon } \\
\hline Bosque abierto & 29 & 120 & 2.8 \\
\hline Tacotal & 26 & 135 & 3.0 \\
\hline Árboles en potreros & 34 & 254 & 3.3 \\
\hline
\end{tabular}




\subsection{Fauna mayor (mamíferos, aves, reptiles) existente en la finca}

Se encontraron 26 especies de aves, 7 de mamíferos y 8 de reptiles, identificadas con la ayuda de baquianos y consulta bibliográfica. Se encontró mayor cantidad en el bosque dado que la masa arbórea es más abundante, por lo tanto, éstas tienen mayor disponibilidad de espacio para hacer nidos, madrigueras, cuevas y además disponen de más alimento y protección. En los otros dos estratos hubo menor cantidad por haber menor abundancia de alimento y más perturbación por la presencia de ganado y personas que hacen ruido durante el pastoreo.

Las aves se encontraron con mayor frecuencia debido a las condiciones de anidamiento y protección que les da el bosque y se observaron con mayor abundancia en el momento de realizar el estudio. De las 26 especies encontradas, 9 son migratorias, las cuales viven en ambientes acuáticos y otras merodean dentro del bosque y áreas arboladas.

Según Fundación del Río, (2013) los humedales de San Miguelito, están conectados con la finca El Morro, siendo esto de gran importancia por ser un espacio de tránsito de la fauna silvestre y de ahí la importancia de conservar la finca, bajo el sistema nacional de áreas protegidas, haciendo una intervención activa para prevenir quemas, corte de árboles, extensión de pastos y caza indiscriminada de la fauna silvestre, estos humedales, han sido declarados sitio RAMSAR, además muchas de las especies de aves que ocupan estos humedales están dentro de la clasificación de los apéndices de CITES, (Cuadro 5).

Cuadro 5. Lista de aves, mamíferos y reptiles encontrados en la finca El Morro y su clasificación CITES.

\begin{tabular}{|c|c|c|c|c|}
\hline $\mathrm{N}^{\circ}$ & Nombre común & Nombre científico & Familia & Apéndice \\
\hline \multicolumn{5}{|c|}{ Aves } \\
\hline 1 & Colibrí (O) & Amazilia rutila Delatre. & Trochilidae & $\|$ \\
\hline 2 & Garza blanca(O) & Ardea alba Linnaeus. & Ardeidae & \\
\hline 3 & Gavilán (O) & Bueto magnirostris Linnaeus. & Accipitridae & $\|$ \\
\hline 4 & Pato aguja (E) & Anhinga anhinga Brisson. & Anhingidae & $\|$ \\
\hline 5 & Golondrina $(O)$ & Hirundo rustica Linnaeu. & Hirundínidae & \\
\hline 6 & Garza morena (O) & Egretta caerulea Linnaeus. & Ardeidae & $\|$ \\
\hline 7 & Urraca (O) & Calocitta formosa Swainson. & Corvidae & \\
\hline 8 & Paloma San Nicolás(O) & Columbina inca Lesson. & Columbidae & \\
\hline 9 & Tórtola gris (E) & Claravis pretiosa Ferrari-Perez. & Columbidae & \\
\hline 10 & Garcilla(O) & Butorides virescens Linnaeus. & Ardeidae & \\
\hline 11 & Chocoyo zapoyol (E) & Brotogeris jugularis Muller. & Psittacidae & $\|$ \\
\hline 12 & Carpintero cabecirrojo(O) & Campephilus guatemalensis Hartlaub. & Picidae & \\
\hline 13 & Martin pescador (O) & Ceryle torquatus, Linnaeus. & Cerylidae & \\
\hline 14 & Zopilote negro $(\mathrm{O})$ & Coragyps atratus Bechistein. & Cathartidae & \\
\hline 15 & $\operatorname{Pijul}(\mathrm{O})$ & Crotophaga sulcirostris Swainson. & Cuculidae & \\
\hline 16 & Piche $(\mathrm{O})$ & Dendrocygna autumnalis Linnaeu. & Anatidae & III \\
\hline 17 & Cigüeñón (E) & Mycteria americana Linnaeu. & Ciconiidae & \\
\hline 18 & Güis (O) & Pitangus sulphuratus Linnaeu. & Tirannidae & \\
\hline 19 & Sargento(E) & Agelaius phoeniceus Linnaeu. & Icteridae & \\
\hline 20 & Oropéndola(O) & Psarocolius montezuma Lesson. & Icteridae & \\
\hline 21 & Gorrión (O) & Passer domesticus Linnaeu. & Passeridae & \\
\hline 22 & Carpintero $(\mathrm{O})$ & Colaptes melanochloros Vigors. & Picidae & \\
\hline 23 & Zanate(O) & Quiscalus nicaraguensis Salvin. & Icteridae & \\
\hline
\end{tabular}




\begin{tabular}{|c|c|c|c|c|}
\hline $\mathrm{N}^{\circ}$ & Nombre común & Nombre científico & Familia & Apéndice \\
\hline 24 & Paloma cola blanca(E) & Columba palumbus Linnaeu. & Columbidae & \\
\hline 25 & Pato chancho (E) & Phalacrocorax brasilianus Brisson. & Phalacrocoracidae & III \\
\hline 26 & Chocoyo cancán(E) & Eupsittula canicularis Linnaeu. & Psittacidae & $\|$ \\
\hline \multicolumn{5}{|c|}{ Mamíferos } \\
\hline 1 & Murciélago vampiro(E) & Desmodus rotundus Geoffroy. & Phyllostomidae & \\
\hline 2 & Mono Congo (E) & Alouatta palliata Gray. & Atelidae & । \\
\hline 3 & Oso perezoso(O) & Choloepus hoffmanni Peters. & Megalonichidae & $\|$ \\
\hline 4 & Guatusa (E) & Dasyprocta punctata Gray. & Dasyproctidae & $\|$ \\
\hline 5 & Armadillo(E) & Dasypus novemcinctus Linnaeu. & Dasypodidae & III \\
\hline 6 & Zorro cola pelada(E) & Didelphis marsupialis & Didelphidae & \\
\hline 7 & Ardilla $(\mathrm{O})$ & Sciurus variegatoides Ogilby. & Sciuridae & III \\
\hline \multicolumn{5}{|c|}{ Reptiles } \\
\hline 1 & Lagartija rayada(E) & Ameiva undulata Wiegmann. & Teiidae & \\
\hline 2 & Terciopelo, barba amarilla $(\mathrm{O})$ & Bothrops asper Garman. & Viperidae & \\
\hline 3 & Cuajipal(E) & Crocodilus sp Linnaeu. & Crocodilidae & \\
\hline 4 & Garrobo negro(O) & Ctenosaura similis Gray. & Iguanidae & \\
\hline 5 & Iguana verde $(\mathrm{O})$ & Iguana iguana Laurenti. & Iguanidae & \\
\hline 6 & Tortuga $(O)$ & Rhinoclemmys funerea Cope. & Geomemydidae & । \\
\hline 7 & Tortuga pintada (E) & Rhinoclemmys pulcherrima Schneider. & Geomemydidae & । \\
\hline 8 & Bejuquilla(O) & Oxibelis aeneus Wagler. & Colubridae & \\
\hline
\end{tabular}

$(\mathrm{O})=$ Corresponde a las especies que se observaron durante la etapa de campo. $(\mathrm{E})=$ Corresponde a las especies referidas por los baquianos y los entrevistados.

\subsection{Calidad del agua en las fuentes hídricas en la finca}

En la finca existen dos nacimientos (ojos de agua), los cuales son utilizados para uso doméstico y para consumo de agua del ganado. En el cuadro 8 se presenta información de estas fuentes hídricas, con los resultados de los análisis químicos.

Cuadro 6. Resultado del análisis de agua de las fuentes hídricas de la finca El Morro, San Miguelito, Río San Juan, agosto, 2013

\begin{tabular}{|c|c|c|c|c|c|c|c|}
\hline \multicolumn{3}{|c|}{ Fuente Hídrica I } & \multicolumn{5}{|c|}{ Latitud $X=729762$ Longitud $Y=058598$} \\
\hline $\mathrm{pH}$ & Nitrito $\mathrm{NO}_{2}$ & $\begin{array}{l}\text { Nitrato } \\
\mathrm{NO}_{3}\end{array}$ & $\begin{array}{l}\text { Amonio } \\
\mathrm{NH}_{4}\end{array}$ & Fosfato & $\begin{array}{l}\text { Dureza de } \\
\text { carbono }\end{array}$ & $\begin{array}{l}\text { Dureza } \\
\text { total }\end{array}$ & Oxígeno \\
\hline 8.5 & $0.2 \mathrm{mg} / \mathrm{l}$ & $1 \mathrm{mg} / \mathrm{l}$ & $0.2 \mathrm{mg} / \mathrm{l}$ & $3 \mathrm{mg} / \mathrm{l}$ & $6 \mathrm{mg} / \mathrm{l}$ & $6 \mathrm{mg} / \mathrm{l}$ & $6 \mathrm{ml} / \mathrm{l}$ \\
\hline \multicolumn{8}{|c|}{ Fuente Hídrica II } \\
\hline 8.2 & $0.3 \mathrm{mg} / \mathrm{l}$ & $2 \mathrm{mg} / \mathrm{l}$ & $0.4 \mathrm{mg} / \mathrm{l}$ & $1 \mathrm{mg} / \mathrm{l}$ & $7 \mathrm{mg} / \mathrm{l}$ & $7 \mathrm{mg} / \mathrm{l}$ & $5 \mathrm{ml} / \mathrm{l}$ \\
\hline
\end{tabular}

\subsection{1. pH}

En general, el pH de la fuente 1 es más alcalino que el de la fuente 2, variando por 3 décimas. Estas fuentes de agua son utilizadas por los habitantes de la finca para lavar ropa, así como para aguar el ganado, estas 
pueden ser las causas de que el $\mathrm{pH}$ sea tan alto, por efecto de detergentes, las heces y orina del ganado, por medio de la precipitación se da arrastre de sedimentos dejando descubierto los suelos, dando inicio al estancamiento del agua.

\subsubsection{Nitritos y nitratos}

Los valores de nitritos encontrados en las fuentes de agua pueden deberse a la descomposición de materia orgánica proveniente de la hojarasca y del arrastre de sedimentos, mientras que las concentraciones de nitratos no sobrepasan el valor máximo admisible de las Normas regionales de calidad del agua para consumo humano que corresponde a $50 \mathrm{mg} / \mathrm{l}$ (CAPRE, 1995). Para este componente el agua de las dos fuentes es admisible para consumo humano.

\subsubsection{Amonio}

El amonio es uno de los subproductos de la descomposición de abonos nitrogenados y de la materia orgánica, una alta concentración de amonio se atribuye a actividades de ganadería y su presencia es indicativa de fuentes contaminantes. En este componente, en ambas fuentes hídricas de la finca, los valores no sobrepasaron el valor de $0.50 \mathrm{mg} / \mathrm{l}$ que establecen las normas CAPRE, para hacer el agua consumible por los humanos.

\subsubsection{Fosfato}

El proceso de contaminación más común resulta de la utilización de fosfatos y nitratos como fertilizantes en los cultivos agrícolas, la descomposición de la materia orgánica de la basura y los detergentes hechos a base de fosfatos, que son arrastrados o arrojados a los ríos (Aguirre y Mendoza, 2014).

Según los niveles de fosfato encontrados en ambas fuentes, éstos se encuentran en el rango establecido en la Norma Técnica Obligatoria Nicaragüense (NTON-05 007-98/2000) de fosfatos para el crecimiento de plantas y animales, el cual oscila entre 1-15 mg/l. Se puede decir, entonces que las fuentes no contienen niveles que afecten o envenenen a las plantas acuáticas o a la fauna.

\subsubsection{Dureza de carbono y dureza del agua}

La dureza del agua está dete rmin ad a po r el contenido de carbonatos, bicarbonatos, cloruros, sulfatos y nitratos de calcio y magnesio; se determina calculando la cantidad de iones $\mathrm{Ca}+2$ y $\mathrm{Mg}+2$ que se encuentran en un volumen medido de agua (Aguirre y Mendoza, 2014).

Diversas publicaciones indican que la dureza no tiene efectos negativos para la salud y el medio ambiente, pero sí provoca inconvenientes tales como la obstrucción de las tuberías debido a que la cal ( $\left.\mathrm{CaCo}_{3}\right)$ se deposita e incrusta sobre las superficies y recipientes con los cuales entra en contacto; asimismo, el uso de aguas duras obliga a utilizar más agua y jabón, ya que impide que se haga espuma, causando atraso y más gasto (Aguirre y Mendoza, 2014). Sin embargo, Mora et al. (2000), citados por (Aguirre y Mendoza, 2014), reportan una relación entre los cálculos en las vías urinarias y su relación con el consumo de calcio en el agua de bebida en Costa Rica, concluyendo que el consumo prolongado de aguas que presentan concentraciones mayores de $120 \mathrm{mg} / \mathrm{l}$ de $\mathrm{CaCO}_{3}$ representa un factor de riesgo para el padecimiento de este tipo de enfermedad.

Para este componente los valores en ambas fuentes fueron iguales, valores que según las normas CAPRE, catalogan el agua como blanda (Cuadro 7) (CAPRE, 1995). Derivado de estos resultados se puede decir que el agua de la finca, desde su caracterización química es apta para consumo humano. Sin embargo, no se puede llegar a una conclusión total, dado que no se hizo análisis biológico de las mismas, por tanto se desconoce la concentración de coliformes fecales en las dos fuentes. 
Cuadro 7. Clasificación según dureza, en relación si es apta para consumo humano

\begin{tabular}{|l|l|}
\hline$B$ & (Blandas): o-60 mg.l \\
\hline$M D$ & (Medianamente dura): 60-120 mg.l \\
\hline$D$ & (Dura): 120-180 mg.l \\
\hline$M / D$ & (Muy dura): mayor de $180 \mathrm{mg} . \mathrm{l}$ \\
\hline
\end{tabular}

\subsubsection{Oxígeno}

El valor que se obtuvo de oxígeno en la fuente I fue de $6 \mathrm{ml} / \mathrm{l}$ y en la en la fuente II fue de $5 \mathrm{ml} / \mathrm{l}$ esto se debe a un mínimo de vegetación en la rivera de las fuentes hídricas.

\subsubsection{Caracterización física del suelo}

En el análisis de laboratorio se obtuvieron los diversos porcentajes de partículas por cada suelo, según el estrato del que provenía, estos porcentajes son reflejados en el cuadro 8. Con base en los porcentajes de partículas y con el uso del triángulo textural de suelo (USDA,1999) se obtuvo que, para el área de bosque franco arcillosos, para el tacotal son suelos arcillosos, y para el área de árboles dispersos en potreros son arcillosos.

Cuadro 8. Caracterización física del suelo en los tres estratos en la finca El Morro.

\begin{tabular}{|c|c|c|c|c|}
\hline \multirow[t]{2}{*}{ ESTRATO } & \multicolumn{3}{|c|}{ PARTíCULAS \% } & \multirow[t]{2}{*}{ CLASE TEXTURA } \\
\hline & Arcilla & Limo & Arena & \\
\hline Tacotal & 45.8 & 34 & 20.2 & Arcilloso \\
\hline Bosque & 39.8 & 36 & 24.2 & Franco-arcilloso \\
\hline Arboles dispersos en potrero & 47.8 & 28 & 24.2 & Arcilloso \\
\hline
\end{tabular}

Según Sampat (1991), los suelos arcillosos poseen baja capacidad de infiltración, baja porosidad, se sobresaturan de agua y son difíciles de manejar. La condición de arcilloso predispone a estos suelos al encharcamiento, lo cual indica que se debe mantener y favorecer el desarrollo de la vegetación natural ya existente en la finca, con el propósito de mitigar el proceso erosivo e inducir a la acumulación de materia orgánica que es la que enriquece los suelos desde el punto de vista de la productividad y además facilita la infiltración del agua.

\subsubsection{Caracterización química de los suelos}

Para la interpretación de las características químicas de los suelos se utilizaron los valores críticos tomados de las normas CAPRE, (1995), se usó esta norma para comparar los valores de la caracterización el cual nos da como referente para determinar si las condiciones del suelo son aptas para el crecimiento de la vegetación todos los valores químicos tal como se observan en el cuadro 11.

En este mismo contexto, el cuadro 10 refleja los resultados de los análisis químicos de las muestras de suelos recolectadas en los tres estratos evaluados en la finca y a partir de los valores críticos del cuadro 9, se hizo la valoración general de los suelos de la finca que aparece en el cuadro 11.

Según las normas CAPRE, los valores encontrados en la finca son permisibles por que la cantidad de arcilla encontrada en los suelos son de valor medio por debajo del valor máximo establecido (cuadro 11). 
Cuadro 9. Valores críticos de los elementos químicos del suelo (Normas CAPRE, 1995).

\begin{tabular}{|l|l|l|l|l|}
\hline & \multicolumn{1}{|c|}{ Baja } & \multicolumn{1}{c|}{ Media } & \multicolumn{1}{c|}{ 0́ptimo } & \multicolumn{1}{c|}{ Alta } \\
\hline $\mathrm{pH}$ & Menor que 5 & De 5 a 6 & De 6 a 7 & Mayores de 7 \\
\hline Ca & Menor que 4 & De 4 a 6 & De 6 a 15 & Mayores de 15 \\
\hline Mg & Menor que 1 & De 1 a 3 & De 3 a 6 & Mayores de 6 \\
\hline K & Menor que 0.2 & De 0.2 a 0.5 & De 0.5 a 0.8 & Mayores de 0.8 \\
\hline P & Menores que 12 & De 12 a 20 & De 20 a 50 & Mayores de 50 \\
\hline Fe & Menores que 5 & De 5 a 10 & De 10 a 50 & Mayores de 50 \\
\hline Cu & Menores que 0.5 & De 0.5 a 1 & De 1 a 20 & Mayores \\
\hline Zn & Menores que 2 & De 2 a 3 & De 3 a 10 & Mayores de 10 \\
\hline Mn & Menores que 5 & De 5 a 10 & De 10 a 50 & Mayores de 50 \\
\hline Mo & Menores que 2 & De 2 a 5 & De 5 a 10 & Mayores de 10 \\
\hline
\end{tabular}

Cuadro 10 Valores de la caracterización química del suelo en los tres estratos en la finca El Morro

\begin{tabular}{|c|c|c|c|c|c|c|c|c|c|c|c|c|c|c|c|}
\hline \multicolumn{6}{|c|}{ Rutina } & \multicolumn{2}{|r|}{ Bases } & \multicolumn{8}{|c|}{ Micro elementos } \\
\hline & $P$ & $\mathrm{MO}$ & $\mathrm{N}$ & disp & K- & $\mathrm{Al}$ & K & $\mathrm{Ca}$ & $\mathrm{Mg}$ & $\mathrm{Na}$ & $\mathrm{ClC}$ & $\mathrm{Fa}$ & $\mathrm{Cu}$ & $\mathrm{Zn}$ & $\mathrm{Mn}$ \\
\hline & $\mathrm{H}_{2} \mathrm{O}$ & $\%$ & & ppm & & & $\begin{array}{l}\text { me/100 g } \\
\text { suelo }\end{array}$ & & & & & ppm & & & \\
\hline Tacotal & 5.43 & 4.35 & 0.22 & 0.89 & & 0.20 & 0.89 & 5.56 & 1.82 & 0.00 & 11.83 & 145.3 & 3.06 & 3.34 & 63.67 \\
\hline Bosque & 5.93 & 5.21 & 0.26 & 1.44 & & & 0.70 & 12.76 & 3.98 & 0.03 & 20.67 & 61.8 & 3.16 & 3.68 & 119.59 \\
\hline \multicolumn{16}{|l|}{ Arboles } \\
\hline $\begin{array}{l}\text { disper- } \\
\text { sos } \\
\text { en po- } \\
\text { treros }\end{array}$ & 5.76 & 5.85 & 0.29 & $\mathrm{Nd}$ & & & 0.20 & 4.54 & 1.88 & 2.78 & 15.21 & 62.5 & 2.79 & 2.89 & 8.32 \\
\hline
\end{tabular}

Cuadro 11. Valoración general de las características de los suelos de la finca El Morro por estrato.

\begin{tabular}{|c|c|c|c|c|c|c|c|c|c|c|c|c|c|c|c|}
\hline \multirow[t]{2}{*}{ Estrato } & \multicolumn{4}{|c|}{ Rutina } & \multicolumn{5}{|c|}{ Bases } & \multicolumn{6}{|c|}{ Micro elementos } \\
\hline & $\mathrm{pH}$ & MO & $\mathrm{N}$ & $\begin{array}{c}\text { P- } \\
\text { disp }\end{array}$ & $\begin{array}{c}\text { K- } \\
\text { disp }\end{array}$ & Al & K & $\mathrm{Ca}$ & $\mathrm{Mg}$ & $\mathrm{Na}$ & $\mathrm{CIC}$ & $\mathrm{Fe}$ & $\mathrm{Cu}$ & $\mathrm{Zn}$ & $\mathrm{Mn}$ \\
\hline & $\mathrm{H}_{2} \mathrm{O}$ & $\%$ & & ppm & & & $\begin{array}{l}\text { me/100 } \\
\text { g suelo }\end{array}$ & & & & & ppm & & & \\
\hline Tacotal & Acido & $M$ & A & B & & B & $A$ & M & M & $\begin{array}{l}\text { No } \\
\text { hay }\end{array}$ & M & $A$ & $\mathrm{O}$ & $\mathrm{O}$ & $A$ \\
\hline Bosque & Acido & 0 & A & B & & & A & M & M & $\begin{array}{l}\text { No } \\
\text { hay }\end{array}$ & M & A & $\mathrm{O}$ & $\mathrm{O}$ & A \\
\hline $\begin{array}{l}\text { Arboles dis- } \\
\text { persos en } \\
\text { potreros }\end{array}$ & Acido & 0 & A & $\mathrm{Nd}$ & & & $A$ & M & M & $\begin{array}{l}\text { No } \\
\text { hay }\end{array}$ & M & A & $\mathrm{O}$ & $\mathrm{O}$ & $A$ \\
\hline
\end{tabular}

MeMedio $\quad \mathrm{O}=$ Optimo $\quad \mathrm{A}=$ =Alto $\quad \mathrm{B}=\mathrm{Bajo} \quad \mathrm{Nd}=$ No disponible 


\section{Conclusiones}

1. Las familias Fabaceae y Mimosaceae son las más representativas en la finca por poseer el mayor número de especies y la familia Dilleniaceae fue la más abundante por poseer el mayor número de individuos.

2. La mayoría de especies no tiene valor comercial y su manejo debe ir dirigido hacia la conservación y renovación de las poblaciones de individuos por especie.

3. Se encontró mayor cantidad de especies de fauna en el estrato bosque abierto por haber mayor cantidad de vegetación que les brinda protección y alimento.

4. En lo referido a la fauna se encontró mayor cantidad de especies de aves que de mamíferos y reptiles, debido a su ubicación próxima al humedal donde trafican aves estacionarias y migratorias.

5. Se encontraron 26 especies de aves, entre ellas, 9 migratorias, 10 especies de mamíferos y 8 especies de reptiles, en los tres tipos de vegetación.

6. Según las normas CAPRE, la calidad del agua se califica como aceptable, es decir que esta puede ser apta para consumo humano, animales domésticos y fauna silvestre. No se puede afirmar lo mismo desde el punto de vista biológico, ya que no se hicieron análisis bacteriológicos.

7. La textura predominante en los suelos es arcillosa y en su composición química predomina la acidez, que limita la actividad agrícola, pero no la conservación de la vegetación.

\section{Agradecimiento}

Esta publicación obtuvo el financiamiento de: El Fondo de Asistencia Internacional de los Estudiantes y Académicos Noruegos (SAIH).

\section{Recomendaciones}

1. Promover la conservación de la finca El Morro con acciones de protección e intervención activa previniendo quemas, corte de árboles, extensión de pastos, la caza indiscriminada de la fauna silvestre y favoreciendo el desarrollo de la vegetación protegiendo la regeneración natural.

2. Realizar estudios de monitoreo y conservación de la biodiversidad existente en la finca.

3. Realizar análisis bacteriológicos en las fuentes de agua para detectar la posible presencia de coliformes fecales y determinar su potabilidad total.

4. Valorar la inclusión de la finca dentro del Sistema Nacional de Áreas Protegidas por estar dentro de los humedales de San Miguelito, sitio RAMSAR 1140.

\section{Lista de referencia}

Aguirre, C. y Mendoza, R. (2014). Informe de monitoreo de calidad del agua para consumo humano, mediante test de campo, en fuentes de comunidades atendidas por COACOV en los municipios Jicaro y Jalapa. Ni. REF. NIC1003-11, pág. 21. 
CAPRE (Comité Coordinador Regional de Instituciones de agua Potable y Saneamiento de Centroamérica, Panamá y República Dominicana, 1995). Norma Regional de Calidad del Agua.

CITES (Convención sobre el comercio internacional de especies amenazadas de fauna y flora Silvestre, 2010). Listado actualizado de las especies de fauna y flora. CITES - USAID. USA 61 P.

Dean, R. (2011). Nicaragua Pacific Slope BIRDS. Quetzali Nicaragua. The guide and the artiare protectod by U.S and international copyright laws. All rights reserved ISBN 978-0-9840207-07.

FUNDACIÓN DEL RIO. (2013). Día mundial de los humedales. EN: EL MANATI (boletín informativo de Río San Juan de Nicaragua) año 7, N.1. Febrero 2013. Rio San Juan de Nicaragua. 4 p.

INETER (2002). Mapa Geológico digitalizado por MARENA a partir de hojas a escala 1:250,000

INIFOM (2010, Instituto Nicaragüense de Fomento Municipal). Ficha municipal: (enlínea) consultadoel22dejulio. 2014 Disponible en http://www.inifom.gob.ni/municipios/documentos/RIO\%20SAN\%20 JUAN/san_miguelito.pdf

MARENA (Ministerio del Ambiente y los Recursos Naturales, NI, 2005). Manejo y aprovechamiento del bosque latifoliado. 1 ed. Managua, Nicaragua. POSAF II. (Programa socioeconómico y desarrollo forestal). 66 pág.

Narváez, O. (2012). Dinámica de crecimiento, estructura y composición de la vegetación secundaria en trópico seco de Nandarola, Nicaragua. Tesis Maestría. Universidad Nacional Agraria. Managua, Nicaragua. 70 p.

NTON (Normas Técnicas Obligatorias Para El Manejo Sostenible De Los Bosque Naturales Latifoliados Y De Coníferas, 2000). Managua, Nicaragua. ICS 18 001-04. Pág. 18

Orozco, L. (1991). Estudios ecológicos y de estructura horizontal de 6 comunidades boscosas de las cordilleras de Talamanca, Costa Rica. INFORAT / CATIE. Informe técnico Nº 179. Turrialba, Costa Rica. 34 pp.

Pla, Laura (2006). Biodiversidad: inferencia basada en el indice de Shannon y la riqueza. Universidad Central de Venezuela. ISSN. 0378-1844. INCI. N.8 v.41. Caracas Venezuela pág. 19.

Ruiz, A. Mariscal, T. (2003). Sitios Ramsar de importancia internacional. Humedales de Nicaragua. Managua. pág. 32

Salas Estrada, Juan B. (1993). Árboles de Nicaragua. Managua, Nicaragua: Instituto Nicaragüense de Recursos Naturales y del Ambiente, IRENA, 1993. 390 pág: il. 117 ils; 9 maps.

Sampat, A. G. (1991). Física de suelo, principios y aplicaciones. Editorial Limusa, México. Pág.17.

USDA. (Departamento de Agricultura de Estados Unidos, 1999). Libro de datos de agricultura. Jul, 2000, pag.315 disponible en http://www.usda.gov 